\title{
Identification of biomarkers of intrahepatic cholangiocarcinoma via integrated analysis of mRNA and miRNA microarray data
}

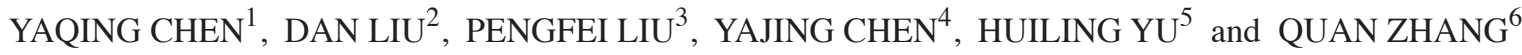 \\ ${ }^{1}$ Department of VIP Ward, Affiliated Hospital of Hebei University, Baoding, Hebei 071000; \\ ${ }^{2}$ Department of Ultrasonic Imaging, Zhuhai People's Hospital, Zhuhai, Guangdong 519000; \\ ${ }^{3}$ Department of Lymphoma, Sino-US Center of Lymphoma and Leukemia, Tianjin Medical University \\ Cancer Institute and Hospital, National Clinical Research Center for Cancer, Key Laboratory of Cancer \\ Prevention and Therapy, Tianjin 300060; ${ }^{4}$ Department of Internal Medicine, Baoding Xiongxian County \\ Hospital; Departments of ${ }^{5}$ Gastroenterology and ${ }^{6}$ Hepatobiliary Surgery, \\ Affiliated Hospital of Hebei University, Baoding, Hebei 071000, P.R. China
}

Received November 6, 2015; Accepted November 7, 2016

DOI: $10.3892 / \mathrm{mmr} .2017 .6123$

\begin{abstract}
The present study aimed to identify potential therapeutic targets of intrahepatic cholangiocarcinoma (ICC) via integrated analysis of gene (transcript version) and microRNA (miRNA/miR) expression. The miRNA microarray dataset GSE32957 contained miRNA expression data from 16 ICC, 7 mixed type of combined hepatocellular-cholangiocarcinoma (CHC), 2 hepatic adenoma, 3 focal nodular hyperplasia (FNH) and 5 healthy liver tissue samples, and 2 cholangiocarcinoma cell lines. In addition, the mRNA microarray dataset GSE32879 contained mRNA expression data from 16 ICC, $7 \mathrm{CHC}, 2$ hepatic adenoma, $5 \mathrm{FNH}$ and 7 healthy liver tissue samples. The datasets were downloaded from the Gene Expression Omnibus database. Differentially expressed genes (DEGs) and miRNAs (DEMs) in ICC samples compared with healthy liver tissues were identified via the limma package, following data preprocessing. Genes that exhibited alternative splicing (AS) in ICC samples were identified via AltAnalyze software. Functional enrichment analysis of DEGs was performed using the Database for Annotation, Visualization and Integrated Analysis. Target genes of DEMs were identified using the TargetScan database. The regulatory association between DEMs and any overlaps among DEGs, alternative splicing genes (ASGs) and target genes of DEMs were retrieved, and a network was visualized using the Cytoscape software. A total of 2,327 DEGs, 70 DEMs and 623 ASGs
\end{abstract}

Correspondence to: Dr Dan Liu, Department of Ultrasonic Imaging, Zhuhai People's Hospital, 79 Kang-Ning Road, Zhuhai, Guangdong 519000, P.R. China

E-mail: danliuhello@163.com

Key words: intrahepatic cholangiocarcinoma, microarray, Gene Expression Omnibus database, Database for Annotation, Visualization and Integrated Analysis were obtained. Functional enrichment analysis indicated that DEGs were primarily enriched in biological processes and pathways associated with cell activity or the immune system. A total of 63 overlaps were obtained among DEGs, ASGs and target genes of DEMs, and a regulation network that contained 243 miRNA-gene regulation pairs was constructed between these overlaps and DEMs. The overlapped genes, including sprouty-related EVH1 domain containing 1, protein phosphate 1 regulatory subunit $12 \mathrm{~A}$, chromosome 20 open reading frame 194, and DEMs, including hsa-miR-96, hsa-miR-1 and $h s a-m i R-25$, may be potential therapeutic targets for the future treatment of ICC.

\section{Introduction}

Intrahepatic cholangiocarcinoma (ICC) is the second most frequently occurring primary liver cancer with a high mortality rate (1), which affects $1-2$ per 100,000 patients (2). Various factors have previously been suggested to contribute to the progression of ICC, including sclerosing cholangitis and hepatobiliary flukes (3). Ultrasound scans may provide diagnostic information (4); however, specific diagnostic criteria for patients with ICC remain to be elucidated. Therefore, numerous patients present with ICC for diagnosis at an advanced stage, which may contribute to its poor prognosis (5). It is therefore important to identify potential biomarkers of ICC to aid prevention and identification of therapeutic strategies.

The progression of ICC is associated with numerous genetic factors, including gene mutations and dysregulation of gene expression (6). Various technological advances have identified molecular targets. Weber et al (7) demonstrated that low frequency alterations of phosphatase and tensin homolog, cyclin-dependent kinase inhibitor 2A, and breast cancer $1 / 2$ may explain the mutation spectrum in ICC, based on clustered regularly interspaced short palindromic repeats (CRISPR)/CRISPR-associated protein 9-based targeted somatic multiplex-mutagenesis. Lee et al (8) profiled the expression of 315 genes and screened 400 alterations in 84 genes, via 
hybridization capture, which may act as therapeutic targets of ICC(8).Variousdrugsortherapeuticmethodshavebeendeveloped based on the aforementioned identified biomarkers. Lovastatin, which is a 3-hydroxy-3-methylglutaryl-coenzyme-CoA reductase inhibitor, may inhibit the proliferation, migration and adhesive activities of ICC cells, by inhibiting the expression of transforming growth factor- $\beta 1$, cyclooxygenase- 2 , and intracellular adhesion molecule-2 (9). Furthermore, various genes have previously been demonstrated to affect drug sensitivity and resistance in ICC. Tepsiri et al (10) demonstrated that the expression of multidrug resistance-associated protein 3 was significantly associated with the half maximal inhibitory concentration of etoposide in ICC. Various biomarkers have been identified; however, the underlying mechanism remains to be fully elucidated, to improve the prognosis and therapy of ICC.

Gene microarrays profile the expression of thousands of genes simultaneously and have been incorporated in numerous cancer and ICC associated investigations (11-13). The present study compared gene and microRNA (miRNA/miR) expression data in ICC samples with healthy liver tissues, and differentially expressed genes (DEGs) and miRNAs (DEMs) were identified. Functional and pathway analyses were conducted, and subsequently, a miRNA-gene regulation network was constructed to explore potential biomarkers of ICC to improve its prognosis.

\section{Materials and methods}

Microarray data. The miRNA expression dataset GSE32957 and mRNA expression dataset GSE32879(14) were downloaded from the Gene Expression Omnibus database (http://www. ncbi.nlm.nih.gov/geo/). A total of 16 ICC, 7 combined hepatocellular-cholangiocarcinoma $(\mathrm{CHC})$ and 2 hepatic adenoma samples were included in the mRNA and miRNA expression datasets. In addition, the miRNA expression dataset contained 3 focal nodular hyperplasia (FNH) and 5 healthy liver tissue, and 2 cholangiocarcinoma cell lines, whereas the mRNA expression dataset contained 5 FNH and 7 healthy liver tissue samples. Hybridizations of miRNA and mRNA were performed on GPL14732 Nanostring nCounter Human microRNA Expression Platform (NanoString Technologies, Inc., Seattle, WA, USA) and GPL6244 [HuGene-1_0-st] Affymetrix Human Gene 1.0 ST Array [transcript (gene) version; Affymetrix, Inc., Santa Clara, CA, USA], respectively.

Differential expression analysis. The free $R$ package NanoStringNorm (15) was used for the data preprocessing of GSE32957, whereas the affy package (https://bioconductor. org/packages/release/bioc/html/affy.html) was selected for analysis of GSE32879, due to the background correction and quartile normalization qualities it exhibits. DEGs and DEMs were identified in ICC samples compared with healthy liver tissues, via the limma package (http://bioconductor.org/packages/release/bioc/html/limma.html) in $R$. DEGs and DEMs were screened with the thresholds of Benjamini-Hochberg adjusted $\mathrm{P}<0.05$ and $\mid \log 2$ (fold change) $\mid>1$.

Alternative splicing (AS) analysis. AS, which is prevalent in the mammalian genome, may generate a complex transcriptome from a finite genome and thus, result in a diverse range of proteins with various functions $(16,17)$. The present study used AltAnalyze (18) software to infer AS via the interpretation of alternative exon inclusion, in ICC samples. Briefly, raw CEL microarray data were normalized using the FIRMA method implemented in AltAnalyze, followed by the identification of alternative splicing genes (ASGs) with all of the default parameters of AltAnalyze. In addition, hierarchical clustering of ASGs in ICC and healthy liver tissue samples were conducted.

Functional and pathway enrichment analysis. The Database for Annotation, Visualization and Integrated Analysis (DAVID; https://david.ncifcrf.gov/) is a widely used web-based tool for functional and pathway enrichment analysis (19). DAVID was used to conduct functional and pathway enrichment analysis of DEGs. $\mathrm{P}<0.05$ was used as the criterion to identify significantly enriched Gene Ontology (GO; http://geneontology.org/) terms and Kyoto Encyclopedia of Genes and Genomes (KEGG; http://www.genome.jp/kegg/) pathways.

Screening of target genes of DEMs. TargetScan (20) (http://www.targetscan.org/) is a web-based software that was used to predict the target genes of DEMs. Overlaps among these target genes, DEGs and ASGs were obtained, and regulatory associations between the overlaps and DEMs were retrieved to construct the miRNA-gene regulation network, which was visualized using Cytoscape software (21).

\section{Results}

Identification of DEGs, DEMs and ASGs. A total of 2,327 DEGs; 1,214 of which were downregulated and 1,113 of which were upregulated, and 70 DEMs, including 5 down- and 65 upregulated, were identified in ICC samples compared with healthy liver tissues. A total of 623 genes exhibited alternative splicing in ICC samples compared with healthy liver tissue samples. Hierarchical clustering of ASGs in ICC and healthy liver tissue samples was conducted, as presented in Fig. 1.

Functional and pathway enrichment analysis of DEGs. Functional and pathway enrichment analysis was performed for the 2,327 DEGs. The majority of enriched GO terms were involved in the activity of the cell, and the most significantly enriched were presented in cell-matrix adhesion. Ten of the GO terms that were randomly selected at $\mathrm{P}<0.05$, are presented in Fig. 2. A total of $51 \mathrm{KEGG}$ pathways associated with metabolism and degradation were revealed to be enriched in DEGs; 10 randomly selected pathways are presented in Table I.

Target genes of DEMs. A total of 956 target genes of DEMs were obtained via TargetScan software (data not shown). In addition, 63 overlaps were identified among these target genes, DEGs and ASGs, and 243 miRNA-gene regulation pairs were retrieved between these overlaps and DEMs. The miRNA-gene regulation network is presented in Fig. 3. Furthermore, 52 miRNA-gene pairs (Table II) exhibited an opposite trend in the alteration of miRNA and gene expression values in ICC samples, compared with healthy liver tissues. 


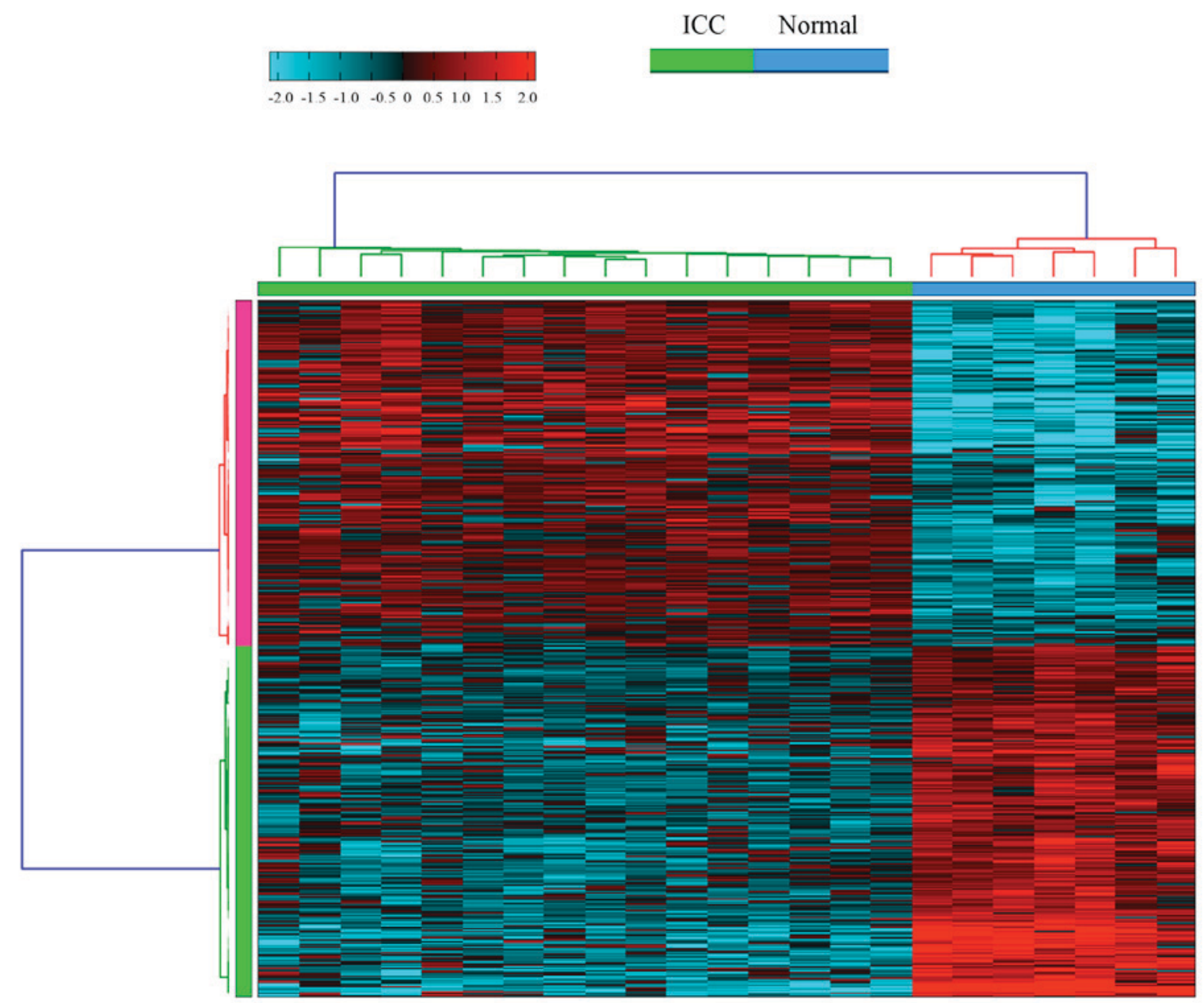

Figure 1. Hierarchical clustering of alternative splicing genes in ICC and healthy liver tissue samples, using AltAnalyze software. The red and blue colors indicate a high and low expression value of alternative splicing genes, respectively. ICC, intrahepatic cholangiocarcinoma.

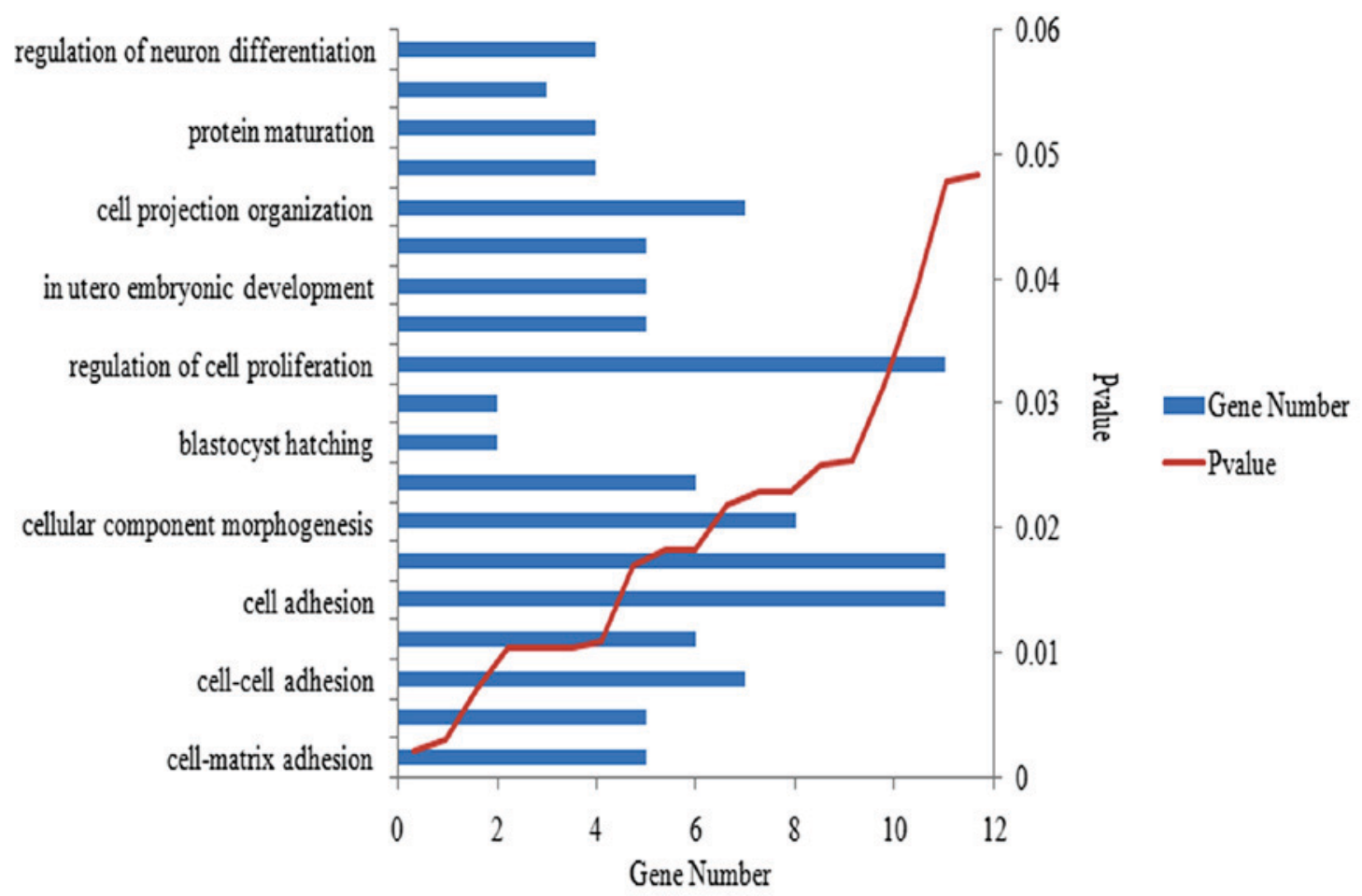

Figure 2. DEG functional and pathway enrichment analysis. Ten GO terms randomly selected from an enrichment analysis of 2327 DEGs using the Database for Annotation, Visualization and Integrated Analysis. P<0.05. DEGs, differently expressed genes. 
Table I. A total of 10 Kyoto Encyclopedia of Genes and Genomes pathways enriched in differentially expressed genes.

\begin{tabular}{lll}
\hline Pathway name & P-value & Gene number \\
\hline hsa04610: Complement and coagulation cascades & $5.92 \mathrm{E}-24$ & 49 \\
hsa00071: Fatty acid metabolism & $1.89 \mathrm{E}-13$ & 28 \\
hsa00260: Glycine, serine and threonine metabolism & $5.08 \mathrm{E}-13$ & 24 \\
hsa00280: Valine, leucine and isoleucine degradation & $5.72 \mathrm{E}-12$ & $1.99 \mathrm{E}-11$ \\
hsa00380: Tryptophan metabolism & $2.63 \mathrm{E}-10$ \\
hsa03320: PPAR signaling pathway & $1.22 \mathrm{E}-09$ & 28 \\
hsa00982: Drug metabolism & $1.10 \mathrm{E}-08$ \\
hsa00330: Arginine and proline metabolism & $1.22 \mathrm{E}-08$ \\
hsa00980: Metabolism of xenobiotics by cytochrome P450 & $7.26 \mathrm{E}-08$ \\
hsa00250: Alanine, aspartate and glutamate metabolism & 34 \\
\hline
\end{tabular}

PPAR, peroxisome proliferator-activated receptor.

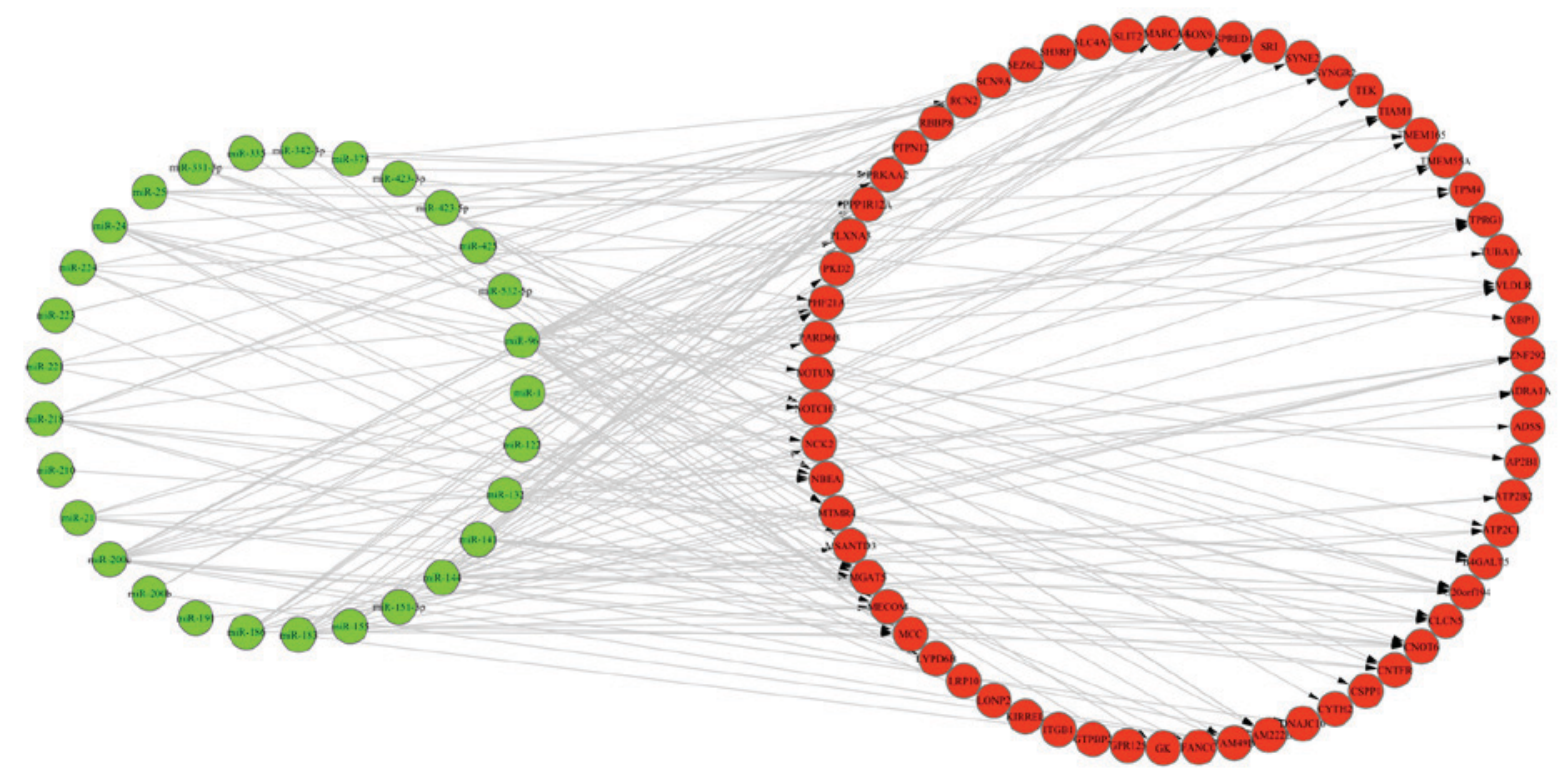

Figure 3. miRNA-gene regulation network. The network was constructed and visualized using Cytoscape software. The green and red circles represent miRNA and genes, respectively. miRNA/miR, microRNA.

\section{Discussion}

In recent years, the emergence of novel diagnostics and treatments, including B-mode ultrasound and chemotherapy, has improved the prognosis of ICC. However, limitations are present in terms of its early diagnosis and cure. The present study conducted a statistical comparison of mRNA and miRNA expression profiles between ICC and healthy liver tissues samples, in order to identify DEGs, DEMs and ASGs. Functional and pathway analyses of DEGs were conducted to explore associated functions and pathways. The miRNA-gene regulation network was constructed to identify the potential biomarkers of ICC.

The GeneChip Human Gene 1.0 ST array (HuGene-1_0-st-v1) is a transcript-based array, used for the detection of gene expression values, and estimation of AS based on the well-annotated exon probes (22). The present study identified the DEGs and
ASGs in ICC samples compared with healthy liver tissues, based on the data detected by HuGene-1_0-st-v1. Functional enrichment analysis indicated that the DEGs were primarily involved in the biological processes associated with cell activity, including cell adhesion and regulation of cell proliferation, which have previously been demonstrated to be associated with cancer progression (23). Pathway enrichment analysis revealed that DEGs were significantly enriched in the pathways associated with substance metabolism and degradation, including fatty acid, glycine, serine and threonine metabolism, and valine, leucine and isoleucine degradation. The extracellular matrix-receptor pathway was also demonstrated to be enriched in DEGs, consistent with the findings of Lee et al (24). The genes that were simultaneously presented in the extracellular matrix-receptor pathway and were revealed to be ASGs, included chondroadherin (CHAD), integrin subunit (ITG)-A3, ITG-B1 and laminin subunit alpha-5 (LAMA5). Three of 
Table II. A total of 52 miRNA-gene pairs presenting opposite trends in the alterations of miRNA and gene expression values in intrahepatic cholangiocarcinoma samples compared with those in healthy liver tissues.

\begin{tabular}{|c|c|}
\hline miRNA & Gene \\
\hline hsa-miR-186 & $A D R A 1 A$ \\
\hline hsa-miR-122 & MGAT5 \\
\hline hsa-miR-132 & $A D R A 1 A$ \\
\hline hsa-miR-96 & TPRG1 \\
\hline hsa-miR-96 & SCN9A \\
\hline hsa-miR-122 & $L R P 10$ \\
\hline hsa-miR-96 & $L O N P 2$ \\
\hline hsa-miR-122 & $A D S S$ \\
\hline hsa-miR-96 & TIAM1 \\
\hline hsa-miR-96 & CLCN5 \\
\hline hsa-miR-96 & $G K$ \\
\hline hsa-miR-200c & TPRG1 \\
\hline hsa-miR-21 & TIAM1 \\
\hline hsa-miR-141 & SCN9A \\
\hline hsa-miR-21 & CNTFR \\
\hline hsa-miR-144 & MGAT5 \\
\hline hsa-miR-218 & TPRG1 \\
\hline hsa-miR-144 & PTPN12 \\
\hline hsa-miR-144 & TMEM165 \\
\hline hsa-miR-200c & CNTFR \\
\hline hsa-miR-141 & TIAM1 \\
\hline hsa-miR-224 & TPRG1 \\
\hline hsa-miR-183 & TIAM1 \\
\hline hsa-miR-141 & CLCN5 \\
\hline hsa-miR-200c & $M C C$ \\
\hline hsa-miR-335 & MTMR4 \\
\hline hsa-miR-144 & ZNF292 \\
\hline hsa-miR-144 & SPREDI \\
\hline hsa-miR-144 & C20orf194 \\
\hline hsa-miR-218 & CLCN5 \\
\hline hsa-miR-1 & GPR125 \\
\hline hsa-miR-223 & $L O N P 2$ \\
\hline hsa-miR-144 & TMEM55A \\
\hline hsa-miR-186 & TPRG1 \\
\hline hsa-miR-155 & CLCN5 \\
\hline hsa-miR-186 & SCN9A \\
\hline hsa-miR-191 & $A T P 2 B 2$ \\
\hline hsa-miR-144 & $V L D L R$ \\
\hline hsa-miR-144 & SMARCA4 \\
\hline hsa-miR-144 & FAM222B \\
\hline hsa-miR-423-5p & CNTFR \\
\hline hsa-miR-218 & $M C C$ \\
\hline hsa-miR-144 & $R C N 2$ \\
\hline hsa-miR-144 & $A T P 2 C 1$ \\
\hline hsa-miR-24 & FANCC \\
\hline hsa-miR-24 & CNTFR \\
\hline hsa-miR-24 & CLCN5 \\
\hline hsa-miR-224 & NOTUM \\
\hline hsa-miR-25 & $X B P 1$ \\
\hline
\end{tabular}

Table II. Continued.

\begin{tabular}{lc}
\hline miRNA & Gene \\
\hline hsa-miR-24 & $M C C$ \\
hsa-miR-532-5p & $M T M R 4$ \\
hsa-miR-186 & $M C C$
\end{tabular}

miRNA/miR, microRNA.

the aforementioned genes, (ITGA3, ITGBI and LAMA5) have previously been demonstrated to be associated with the progression of ICC $(25,26)$. CHAD encodes a cartilage matrix protein, which may mediate adhesion of isolated chondrocytes. CHAD may be included in the phosphoinositide 3-kinase-Akt and focal adhesion signaling pathways, and may be regulated by $\mathrm{p} 53$, which is associated with the pathogenesis and development of numerous cancers, including ICC (http://www. genecards.org). Therefore, $C H A D$ may be a novel biomarker of ICC that may contribute to its progression; however, further studies are required to confirm this.

miRNA are non-coding RNA molecules, which contain $\sim 22$ nucleotides, and are present in animals, plants and viral genomes. miRNAs may induce RNA-silencing and regulate gene expression at the post-transcriptional stage $(27,28)$. The dysregulation of miRNA may result in numerous diseases, including ICC (29-31). The present study identified DEMs in ICC samples compared with healthy liver tissues samples, and the screening of DEM target genes was conducted using the TargetScan database. A miRNA-gene regulation network was constructed based on the DEMs and the overlapping genes among the DEGs, ASGs and target genes of the DEMs. In the regulation network, $h s a-m i R-96$ regulated 22 target genes and 6 out of these regulation pairs [hsa-miR-96-tumor protein P63 regulated 1, hsa-miR-96-sodium voltage-gated channel alpha subunit 9, hsa-miR-96-lon peptidase 2, peroxisomal, $h s a-m i R-96$-T-cell lymphoma invasion and metastasis 1 (TIAM1), hsa-miR-96-chloride voltage-gated channel 5, hsa-miR-96-glycerol kinase] revealed an opposite trend in the alterations of miRNA and mRNA expression values (upregulated miRNA and downregulated gene expression levels). hsa-miR-96 is closely associated with cell proliferation and growth, and Collins et al (32) demonstrated that $h s a-m i R-96$ may be used to identify cholangiocarcinoma and pancreatic adenocarcinoma. Furthermore, the dysregulation of $h s a-m i R-96$ was revealed to contribute to the progression of ICC (33). TIAMI is a target gene of hsa-miR-96, which is important in cellular migration and remodeling of the actin cytoskeleton, and may be involved in the proliferation and migration of ICC via effects on RasGEF domain family member 1A expression (34). Further target genes of $h s a-m i R-96$ have previously been identified, and may act as novel biomarkers of ICC, however further experimental verification is necessary (35).

In conclusion, the present study identified DEGs, ASGs and DEMs in ICC samples compared with healthy liver tissues, via bioinformatics analysis of miRNA and mRNA expression data. Functional and pathway analyses indicated that DEGs 
were primarily involved in biological processes or pathways associated with cancer progression or substance metabolism. A miRNA-gene regulation network was constructed based on DEMs and overlaps among DEGs, ASGs and target genes of DEMs. Furthermore, biomarkers that have previously been identified, including $h s a-m i R-96$ and TIAM1, and numerous novel biomarkers in ICC, including anthranilate synthase component II and sodium voltage-gated channel a subunit 9 , were identified. However, the functions of these biomarkers in ICC remain to be elucidated.

\section{References}

1. Guglielmi A, Ruzzenente A, Campagnaro T, Pachera S, Valdegamberi A, Nicoli P, Cappellani A, Malfermoni G and Iacono C: Intrahepatic cholangiocarcinoma: Prognostic factors after surgical resection. World J Surg 33: 1247-1254, 2009.

2. Anderson CD, Pinson CW, Berlin J and Chari RS: Diagnosis and treatment of cholangiocarcinoma. Oncologist 9: 43-57, 2004.

3. Vilana R, Forner A, Bianchi L, García-Criado A, Rimola J, de Lope CR, Reig M, Ayuso C, Brú C and Bruix J: Intrahepatic peripheral cholangiocarcinoma in cirrhosis patients may display a vascular pattern similar to hepatocellular carcinoma on contrast-enhanced ultrasound. Hepatology 51: 2020-2029, 2010.

4. Khan SA, Thomas HC, Davidson BR and Taylor-Robinson SD: Cholangiocarcinoma. Lancet 366: 1303-1314, 2005.

5. Khan SA, Davidson BR, Goldin R, Pereira SP, Rosenberg WM, Taylor-Robinson SD, Thillainayagam AV, Thomas HC, Thursz MR and Wasan H; British Society of Gastroenterology: Guidelines for the diagnosis and treatment of cholangiocarcinoma: Consensus document. Gut 51: (Suppl 6) VI1-VI9, 2002.

6. Isa T, Tomita S, Nakachi A, Miyazato H, Shimoji H, Kusano T, Muto Y and Furukawa M: Analysis of microsatellite instability, K-ras gene mutation and $\mathrm{p} 53$ protein overexpression in intrahepatic cholangiocarcinoma. Hepatogastroenterology 49: 604-608, 2002.

7. Weber J, Ollinger R, Friedrich M, Ehmer U, Barenboim M, Steiger K, Heid I, Mueller S, Maresch R, Engleitner T, et al: CRISPR/Cas 9 somatic multiplex-mutagenesis for high-throughput functional cancer genomics in mice. Proc Natl Acad Sci USA 112: 13982-13987, 2015

8. Lee H, Wang K, Johnson A, Jones DM, Ali SM, Elvin JA, Yelensky R, Lipson D, Miller VA, Stephens PJ, et al: Comprehensive genomic profiling of extrahepatic cholangiocarcinoma reveals a long tail of therapeutic targets. J Clin Pathol 69: 403-408, 2016

9. Yang SH, Lin HY, Changou CA, Chen CH, Liu YR, Wang J, Jiang $X$, Luh $F$ and Yen Y: Integrin $\beta 3$ and LKB1 are independently involved in the inhibition of proliferation by lovastatin in human intrahepatic cholangiocarcinoma. Oncotarget 7: 362-373, 2016.

10. Tepsiri N, Chaturat L, Sripa B, Namwat W, Wongkham S, Bhudhisawasdi V and Tassaneeyakul W: Drug sensitivity and drug resistance profiles of human intrahepatic cholangiocarcinoma cell lines. World J Gastroenterol 11: 2748-2753, 2005.

11. Zhang MY, Li SH, Huang GL, Lin GH, Shuang ZY, Lao XM, $\mathrm{Xu}$ L, Lin XJ, Wang HY and Li SP: Identification of a novel microRNA signature associated with intrahepatic cholangiocarcinoma (ICC) patient prognosis. BMC Cancer 15: 64, 2015.

12. Wang J, Xie H, Ling Q, Lu D, Lv Z, Zhuang R, Liu Z, Wei X, Zhou L, Xu X and Zheng S: Coding-noncoding gene expression in intrahepatic cholangiocarcinoma. Transl Res 168: 107-121, 2016.

13. Xu YF, Ge FJ, Han B, Yang XQ, Su H, Zhao AC, Zhao MH, Yang YB and Yang J: High-mobility group box 1 expression and lymph node metastasis in intrahepatic cholangiocarcinoma. World J Gastroenterol 21: 3256-3265, 2015.

14. Oishi N, Kumar MR, Roessler S, Ji J, Forgues M, Budhu A, Zhao X, Andersen JB, Ye QH, Jia HL, et al: Transcriptomic profiling reveals hepatic stem-like gene signatures and interplay of miR-200c and epithelial-mesenchymal transition in intrahepatic cholangiocarcinoma. Hepatology 56: 1792-1803, 2012.
15. Waggott D, Chu K, Yin S, Wouters BG, Liu FF and Boutros PC: NanoStringNorm: An extensible $\mathrm{R}$ package for the pre-processing of NanoString mRNA and miRNA data. Bioinformatics 28: 1546-1548, 2012

16. Graveley BR: Alternative splicing: Increasing diversity in the proteomic world. Trends Genet 17: 100-107, 2001.

17. Cieply B and Carstens RP: Functional roles of alternative splicing factors in human disease. Wiley Interdiscip Rev RNA 6: 311-326, 2015.

18. Emig D, Salomonis N, Baumbach J, Lengauer T, Conklin BR and Albrecht M: AltAnalyze and DomainGraph: Analyzing and visualizing exon expression data. Nucleic Acids Res 38: W755-W762, 2010.

19. Dennis G Jr, Sherman BT, Hosack DA, Yang J, Gao W, Lane HC and Lempicki RA: DAVID: Database for Annotation, Visualization, and Integrated Discovery. Genome Biol 4: P3, 2003.

20. Agarwal V, Bell GW, Nam JW and Bartel DP: Predicting effective microRNA target sites in mammalian mRNAs. Elife 4: 2015.

21. Shannon P, Markiel A, Ozier O, Baliga NS, Wang JT, Ramage D, Amin N, Schwikowski B and Ideker T: Cytoscape: A software environment for integrated models of biomolecular interaction networks. Genome Res 13: 2498-2504, 2003.

22. Pradervand S, Paillusson A, Thomas J, Weber J, Wirapati P, Hagenbüchle $\mathrm{O}$ and Harshman K: Affymetrix Whole-Transcript Human Gene 1.0 ST array is highly concordant with standard 3' expression arrays. Biotechniques 44: 759-762, 2008.

23. Sawai H, Okada Y, Funahashi H, Matsuo Y, Takahashi H, Takeyama $\mathrm{H}$ and Manabe T: Activation of focal adhesion kinase enhances the adhesion and invasion of pancreatic cancer cells via extracellular signal-regulated kinase-1/2 signaling pathway activation. Mol Cancer 4: 37, 2005.

24. Lee JI and Campbell JS: Role of desmoplasia in cholangiocarcinoma and hepatocellular carcinoma. J Hepatol 61: 432-434, 2014.

25. Utispan K, Thuwajit P, Abiko Y, Charngkaew K, Paupairoj A, Chau-in S and Thuwajit C: Gene expression profiling of cholangiocarcinoma-derived fibroblast reveals alterations related to tumor progression and indicates periostin as a poor prognostic marker. Mol Cancer 9: 13, 2010.

26. Soejima Y, Inoue M, Takahashi Y, Uozaki H, Sawabe M and Fukusato T: Integrins $\alpha v \beta 6, \alpha 6 \beta 4$ and $\alpha 3 \beta 1$ are down-regulated in cholangiolocellular carcinoma but not cholangiocarcinoma. Hepatol Res 44: E320-E334, 2014.

27. Ambros V: The functions of animal microRNAs. Nature 431: 350-355, 2004

28. Bartel DP: MicroRNAs: Genomics, biogenesis, mechanism, and function. Cell 116: 281-297, 2004.

29. Sun B, Xie C, Zheng T, Yin D, Wang J, Liang Y, Li Y, Yang G, Shi H, Pei T, et al: Selecting molecular therapeutic drug targets based on the expression profiles of intrahepatic cholangiocarcinomas and miRNA-mRNA regulatory networks. Oncol Rep 35: 382-390, 2016.

30. Deng G, Teng Y, Huang F, Nie W, Zhu L, Huang W and Xu H: MicroRNA-101 inhibits the migration and invasion of intrahepatic cholangiocarcinoma cells via direct suppression of vascular endothelial growth factor-C. Mol Med Rep 12: 7079-7085, 2015

31. Xiong X, Sun D, Chai H, Shan W, Yu Y, Pu L and Cheng F: MiR-145 functions as a tumor suppressor targeting NUAK1 in human intrahepatic cholangiocarcinoma. Biochem Biophys Res Commun 465: 262-269, 2015

32. Collins AL, Wojcik S, Liu J, Frankel WL, Alder H, Yu L, Schmittgen TD, Croce CM and Bloomston M: A differential microRNA profile distinguishes cholangiocarcinoma from pancreatic adenocarcinoma. Ann Surg Oncol 21: 133-138, 2014.

33. Papaconstantinou I, Karakatsanis A, Gazouli M, Polymeneas G and Voros D: The role of microRNAs in liver cancer. Eur J Gastroenterol Hepatol 24: 223-228, 2012.

34. Ura K, Obama K, Satoh S, Sakai Y, Nakamura Y and Furukawa Y: Enhanced RASGEF1A expression is involved in the growth and migration of intrahepatic cholangiocarcinoma. Clin Cancer Res 12: 6611-6616, 2006.

35. Chang CC, Lin CC, Hsieh WL, Lai HW, Tsai CH and Cheng YW: MicroRNA expression profiling in PBMCs: A potential diagnostic biomarker of chronic hepatitis C. Dis Markers 2014: 367157, 2014 